\title{
Poor encoding of position by contrast-defined motion
}

\author{
Harriet A. Allen ${ }^{\mathrm{a}, *}$, Tim Ledgeway ${ }^{\mathrm{b}}$, Robert F. Hess ${ }^{\mathrm{a}}$ \\ ${ }^{a}$ McGill Vision Research Unit, 687 Pine Avenue West, Rm. H4-14, Montreal, Que., Canada H3A 1 A1 \\ b School of Psychology, University of Nottingham, University Park, Nottingham NG7 2RD, UK
}

Received 17 November 2003; received in revised form 19 March 2004

\section{Abstract}

Second-order (contrast-defined) motion stimuli lead to poor performance on a number of tasks, including discriminating form from motion and visual search. To investigate this deficiency, we tested the ability of human observers to monitor multiple regions for motion, to code the relative positions of shapes defined by motion, and to simultaneously encode motion direction and location. Performance with shapes from contrast-defined motion was compared with that obtained from luminance-defined (first-order) stimuli. When the position of coherent motion was uncertain, direction-discrimination thresholds were elevated similarly for both luminance-defined and contrast-defined motion, compared to when the stimulus location was known. The motion of both luminance- and contrast-defined structure can be monitored in multiple visual field locations. Only under conditions that greatly advantaged contrast-defined motion, were observers able to discriminate the positional offset of shapes defined by either type of motion. When shapes from contrast-defined and luminance-defined motion were presented under comparable conditions, the positional accuracy of contrast-defined motion was found to be poorer than its luminance-defined counterpart. These results may explain some, but possibly not all, of the deficits found previously with second-order motion.

(C) 2004 Published by Elsevier Ltd.

Keywords: Second-order motion; First-order motion; Position; Direction

\section{Introduction}

Most objects in the visual world are defined by changes in luminance (brightness) over space. The motion of these objects is correlated with a change in luminance over time and space and is often termed 'firstorder' motion (Cavanagh \& Mather, 1989). Objects and motion can also be defined by changes in other visual characteristics, such as changes in texture type, element size or element contrast. These patterns are often termed 'second-order' (Cavanagh \& Mather, 1989). This paper is concerned with one type of 'second-order' moving pattern-moving contrast-defined patterns.

\subsection{Failures with second-order motion}

There are several tasks that have been found to be difficult, or impossible, with moving contrast-defined patterns. Observers are unable to find a patch of con-

\footnotetext{
${ }^{*}$ Corresponding author. Present address: School of Psychology, University of Birmingham, Edgbaston, Birmingham B15 2TT, UK.

E-mail address: h.a.allen@bham.ac.uk (H.A. Allen).
}

trast-defined structure moving in one direction when it is surrounded by patches of contrast-defined structure moving in another direction. This is the case when the motion areas are abutting, creating a surface (Dosher, Landy, \& Sperling, 1989), when they are arranged in a visual search display (Ashida, Seiffert, \& Osaka, 2001), when they define three-dimensional shape (Ziegler \& Hess, 1999) or form a global optic flow pattern (Allen \& Derrington, 2000). These failures might indicate that judging the direction of contrast-defined motion may only be possible at one location in the visual field at a time, for example, because second-order motion perception is mediated primarily by an attention-driven process. Another possibility is that even though multiple estimates of second-order motion can be made across the visual field, individual detectors are poorly labeled for location.

Consistent with the idea that attention is required to discriminate the direction of contrast-defined motion Lu, Liu, and Dosher (2000) found that attention enhances observers' performance when they discriminate the direction of contrast-defined motion. In their study, observers made successive judgments of the directions of 
motion in two, spatially distinct, patches. They found that observers were better able to discriminate the direction of contrast-defined motion in the attended patch, compared to the unattended patch. When the patches contained first-order, luminance-defined, motion, there was no difference between observers' performance with the two patches. Lu et al. (2000) proposed that attention enhances the processing of contrast-defined motion, however this does not necessarily mean that attention is always required for processing of contrast-defined motion.

When attention is distracted, by a distracter task, from contrast-defined motion, performance does not decrease compared to when the same task is performed without a distracter task (Allen \& Derrington, 2001; Ho, 1998). Furthermore, Allen and Ledgeway (2003) found that although they could replicate the different performance with attended and unattended contrast-defined motion reported by $\mathrm{Lu}$ et al. (2000), the magnitude of the attentional enhancement found depended critically on the speed and duration of the stimuli used. These results taken together suggest that, as with many tasks, attending to the stimulus may help observers when sensitivity to the stimulus is low, but attention is not always a necessary requirement for processing secondorder motion.

An alternative explanation for observers' poor performance on certain tasks with second-order motion is that the position of contrast-defined motion is not encoded with great precision. If the encoded position of motion is poorly specified, it could compromise the fidelity with which this motion could be used to determine three-dimensional shape based on motion cues alone. In a search display, if the ability to accurately locate the positions of the motion elements is relatively impoverished, it might also be difficult to discriminate an odd motion, since motion direction is typically dependant on position in experiments of this kind (Allen $\&$ Derrington, 2000). This study was designed to directly investigate how well the human visual system is able to discriminate the position or location of contrast-defined motion.

\subsection{Locating second-order structure}

Although no studies have directly investigated the ability of observers to identify the location of secondorder motion, there have been some studies addressing the ability of observers to identify the location of both static contrast-defined form and another second-order stimulus: motion-defined form.

The mechanism that processes static contrast-defined form seems similar in its ability to localize an object (or border) to the mechanism that processes luminance-defined form. Although localization of contrast-modulations is worse than for luminance-modulated patterns, it can be explicable in terms of gross differences in stimulus complexity or spectral content and is nonetheless in the hyperacuity range (Voltz \& Zanker, 1996). As with firstorder patterns, the perceived location of contrast-modulations can be predicted by the position of their centroids (Whitaker, McGraw, Pacey, \& Barrett, 1996). Adapting to a static stimulus can influence the perceived position of a subsequently viewed pattern (McGraw, Levi, \& Whitaker, 1999; Whitaker, McGraw, \& Levi, 1997) and this is the case for both luminance-defined and contrast-defined patterns, suggesting that similar mechanisms process the two types of pattern. Results from contrast-defined static form have not always, however, generalized to moving contrast-defined patterns. Long presentation durations are required to discriminate the direction of some moving contrast-defined patterns (Derrington, Badcock, \& Henning, 1993) whereas static contrast-modulations are visible at short durations (Cropper, 1998; Schofield \& Georgeson, 2000).

The ability of observers to discriminate the position of one sort of form from a second-order cue, namely motion-defined form, has also been studied. Observers are able to discriminate a Vernier offset between two motion-defined rectangles with fairly high precision (Regan, 1986). Vernier acuity for motion-defined form can match that found with luminance-defined form if the perceptual quality (e.g. perceived contrast) is matched between the two types of stimulus (Banton \& Levi, 1993). Furthermore, motion-defined forms can be compared over space with similar accuracy as that for luminance-defined forms (Kohly \& Regan, 2002). Thus it is clear that there is some mechanism able to identify the location of motion-defined form.

It is often assumed that all forms of second-order stimuli are processed equivalently. Form-cue invariant neurons have been found in the medial-temporal area of the rhesus monkey (Albright, 1992). These respond to flicker-defined forms as well as luminance-defined patterns. This cue-invariance does not seem to generalize to motion-defined forms (Churan \& Ilg, 2001). In behavioural and psychophysical studies performance with different forms of second-order motion is often similar, but not identical. Both contrast-defined motion and flicker-defined motion lead to slow, inefficient search performance, but response times to flicker-defined motion are much faster than those to contrastdefined motion (Ashida et al., 2001). Whilst the direction of moving contrast-modulations can be discriminated in the periphery (Smith \& Ledgeway, 1998) the direction of moving flicker-defined bars cannot be resolved in the periphery (McCarthy, Pantle, \& Pinkus, 1994) even though the bars can be detected. At the very least, different forms of second-order moving patterns must be processed by different processes at the earliest stages of processing. This may lead to different proper- 
ties at later stages of processing. Furthermore, moving contrast-defined patterns combine both motion-defined form and contrast-defined cues, if all second-order motion is processed (eventually) by a common mechanism, one might expect that combining these cues might advantage performance. On the other hand, if contrastdefined form and motion-defined form are resolved at different places in the visual stream performance might be disadvantaged, for example, contrast-defined form might be resolved late in the visual stream, and not be available to the processes that resolve relative motion.

It seems that the relative location of an item can be accurately determined when it is defined by luminance, contrast or relative motion. The aim of this study was to investigate if the location of form defined by moving contrast-defined structure can also be discriminated with a similar degree of efficacy.

\subsection{Spatial uncertainty}

Since we wanted to investigate location discrimination in relation to direction discrimination, it was necessary to also simultaneously measure directiondiscrimination performance. This task is essentially a motion-discrimination task under cued and uncued spatial location conditions, similar to those that have been used to investigate mechanisms of attention. This allowed us to also investigate whether the deficits associated with second-order motion stimuli are due to an inability to simultaneously monitor multiple locations across the visual field.

When observers have to find a patch containing contrast-defined motion moving in an inconsistent direction to the global pattern, their performance is consistent with a slow, patch by patch search of the display (Allen \& Derrington, 2000). The duration required to find the inconsistent motion depends on the number of possible positions of the motion patch. The same task is quick, easy and not dependent on the number of possible positions with moving luminancedefined patterns. This could indicate that positional uncertainty selectively disadvantages the mechanisms that process contrast-defined motion.

When spatial uncertainty is reduced, for example by cueing the location of the stimulus, sensitivity typically improves. This can be attributed to a change in the way a mechanism responds to the stimulus (e.g. Carrasco, Penpeci-Talgar, \& Eckstein, 2000), often termed stimulus enhancement. The improvement in performance can also be attributed to a change in the number of locations or channels that a hypothesized decision process monitors (e.g. Foley \& Schwarz, 1998, see this reference for a review).

In a different task, where observers had to report the direction of motion in two locations, but without specifically manipulating spatial uncertainty, $\mathrm{Lu}$ et al.
(2000) found results consistent with signal enhancement for contrast-defined motion in the attended location, but no such signal enhancement for first-order motion. If manipulating (e.g. reducing) spatial uncertainty also leads to signal enhancement, we would expect a greater effect for second-order motion. Similarly, if manipulating spatial uncertainty changes the number of locations that need to be monitored, and observers are worse at monitoring multiple locations for second-order motion, we would also expect a greater effect of spatial cueing for second-order motion.

\subsection{Three location/position tasks}

We carried out three experiments. First we measured direction-discrimination performance both with and without spatial uncertainty regarding the position of the motion. Second, we measured observers' ability to discriminate whether a motion-defined form was to the left or right of two reference cues. Results from pilot experiments suggested that observers were unable to do this task with many examples of contrast-defined motion. We ran extensive pilot investigations to find a set of parameters for which we were able to estimate relative position thresholds. We collected data for contrast-defined stimuli at different modulation depths, with cue squares defined by moving and static dots, with and without a carrier in the background of the stimulus, with different densities of dots, different speeds and different viewing distances. In all cases, position discrimination was poor and in most cases performance was at chance. Finally we measured the ability of observers to discriminate the absolute location of form conveyed by luminance-defined and contrast-defined motion stimuli supporting comparable (i.e. relative to threshold) levels of performance.

\section{Methods}

\subsection{Observers}

There were four observers, all had normal or corrected-to-normal vision and were experienced participants in psychophysical tasks. Observer HA was one of the authors, observers JD, NK and $\mathrm{PH}$ were naïve to the purposes of the experiment.

\subsection{Apparatus}

The stimuli were presented on a Sony Trinitron Multiscan 520GS monitor with a mean luminance of 41 $\mathrm{cd} / \mathrm{m}^{2}$ and a frame refresh rate of $100 \mathrm{~Hz}$. One screen pixel extended $0.3 \mathrm{~mm}$ horizontally and vertically. Prior to the experiment the relationship between the voltage input to the monitor and the screen luminance was lin- 

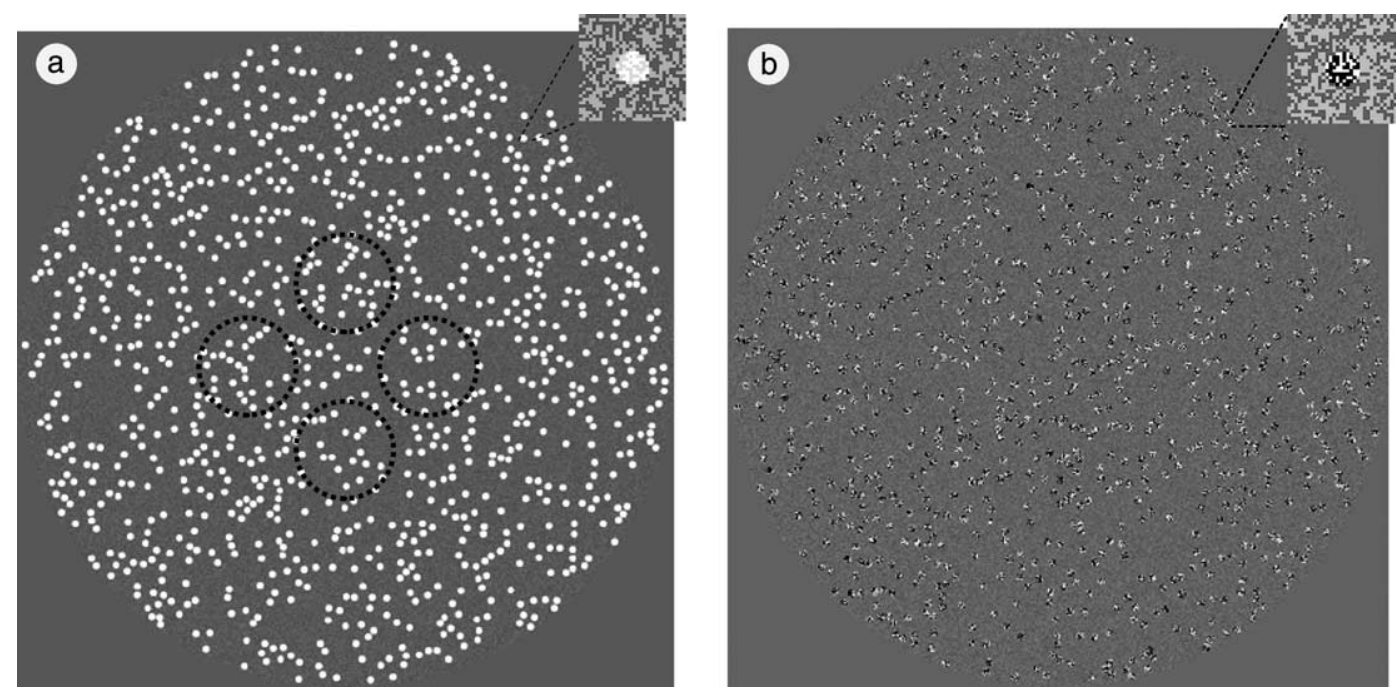

Fig. 1. (a) First-order (luminance-defined) dots as used in Experiment 1 (and also Experiment 3). The dotted circles illustrate the positions of the possible target areas defined by coherent motion (the dotted outlines of the circles were not presented in the actual experiments). (b) Second-order (contrast-defined) dots at maximum modulation depth as used in Experiment 1 (and Experiment 3). Insets to (a) and (b) show a magnified view illustrating the detailed structure of a single dot.

earised (gamma corrected) using a UDT S370 photometer and look-up-tables. The adequacy of the applied gamma correction was also confirmed using a sensitive psychophysical nulling task (Ledgeway \& Smith, 1994; Nishida, Ledgeway, \& Edwards, 1997).

\section{Experiment 1}

In experiment 1 the observers judged the direction of motion in a patch containing coherently moving dots that was positioned in one of four locations. Performance was compared when the observers had prior knowledge of the position of the coherent motion and when they did not have this knowledge. This experiment was designed to measure the effect of positional uncertainty on the ability of observers to discriminate the direction of motion and whether observers can monitor multiple locations over the visual field for motion direction.

\subsection{Stimuli}

Stimuli were presented within a circular display window (aperture) that subtended $14.8^{\circ}$ (diameter) of visual angle at a viewing distance of $97.8 \mathrm{~cm}$. The remainder of the screen was at mean luminance. A central fixation point that appeared immediately before and after each stimulus was presented in order to minimize ocular tracking and maintain stable fixation.

The stimuli were moving circular dots presented on a low contrast, two-dimensional (2-d), binary, static noise background (carrier). The background noise had a Michelson contrast of 0.1 . Luminance-modulated dots or contrast-modulated dots (794) were presented on this noise background. Dots were 10 pixels in diameter. To generate luminance-modulated dots the mean luminance of the noise (both 'dark' and 'light' elements) was increased within the circular region bounding each dot (see below). To generate the contrast-modulated dots the contrast of the noise elements was increased within the circular region bounding each dot. Fig. 1 shows example frames of first-order dots at high contrast (1a) and second-order dots at maximum modulation depth (1b).

The duration of the motion sequence was either 250 or $100 \mathrm{~ms}$. Motion sequences were constructed by displacing the dots by 7 pixels every $50 \mathrm{~ms}$ for the long duration stimulus and by 3 pixels every $20 \mathrm{~ms}$ for the short duration stimulus, giving the dots in each case a speed of $3 \%$. The direction of motion of each dot was independently determined on each displacement depending on whether that dot belonged to the population of dots that were required to move coherently ('signal' dots moving either upwards or downwards on each trial) or randomly ('noise' dots) and whether or not the dot was inside the area of the display containing the patch of coherent motion to be judged by the observer.

Dots in the background area always moved in a random direction on each jump (i.e. were 'noise dots'). On each trial an area was defined as the area of coherent motion, termed for convenience, the target area. The dots within this area moved either up or down with various levels of coherence (i.e. contained a proportion of 'signal' to 'noise' dots so that the signal:noise ratio could be varied). The target area was circular, its radius was $0.9^{\circ}$ and its center was $1.7^{\circ}$ from the center of the display area. It could be in one of four positions, either
301

302

303

304

305

306

307

308

309

310

311

312

313

314

315

316

317

318

319

320

321

322

323

324

325

326

327

328

329

330

331

332

333

334 
directly above, below, left or right of the center of the display area (as illustrated in Fig. 1). When the observer had prior knowledge of the position of the target area containing coherent motion, this position remained the same throughout all the trials of a run. When the observer did not have prior knowledge of the location of motion the position of the target area was randomly selected, on each trial, from the four possible positions. Throughout the experiment the observers fixated the center of the stimulus area.

It is important to note that there were no spatial density differences between the target area and remainder of the display which observers could use to identify the location of the target area (the target area differed only from the background in that it contained a proportion of dots that underwent some degree of coherent, unidirectional motion). Whenever a dot was displaced such that it would fall outside the target area it was immediately replotted within the area at the diagrammatically opposite location. Thus even when there was a high level of motion coherence there were no spatial dot density cues available that could be used to locate the target patch.

\subsection{Procedure}

A single interval, 2-Alternative-Forced-Choice (2AFC) procedure was employed. On each trial observers were presented with a central fixation point followed by a motion stimulus. After the presentation of the stimulus, observers indicated with a key press whether they saw upwards or downwards motion. Motion coherence within the target area (or dot visibility, see below) was controlled by a 1-up 3-down staircase that converged on a threshold corresponding to a performance level of $79 \%$ correct. The staircase terminated after eight reversals and the threshold was taken as the mean of the last six reversals. For each condition tested, 10 staircases were completed and the data point for that condition was taken as the mean of the 10 staircase threshold estimates.

\subsection{Modulation-depth thresholds}

In this and the following experiments, first-order dots were (unless otherwise specified) luminance-modulations (LM) of a spatially 2-d, binary, noise field, such that the luminance of the noise within each dot was higher than that of the background. The dot luminance-modulation depth (dot contrast) was defined as:

Luminance-modulation depth

$$
=\left(D_{\mathrm{L}}-B_{\mathrm{L}}\right) /\left(D_{\mathrm{L}}+B_{\mathrm{L}}\right)
$$

where $D_{\mathrm{L}}$ and $B_{\mathrm{L}}$ are the mean luminances of the 2-d noise (carrier) comprising the dots and the background, respectively. Second-order dots were contrast-modula- tions $(\mathrm{CM})$ of 2-d noise, with higher contrast than the background. The dot contrast-modulation depth was defined as:

Contrast-modulation depth $=\left(D_{\mathrm{c}}-B_{\mathrm{c}}\right) /\left(D_{\mathrm{c}}+B_{\mathrm{c}}\right)$

where $D_{\mathrm{c}}$ and $B_{\mathrm{c}}$ are the mean contrasts of the 2-d noise within the dots and the background, respectively.

Modulation-depth thresholds were measured separately for each observer. On each trial, all of the dots within the target area moved either up or down with $100 \%$ coherence. The staircase controlled the luminancemodulation depth (for first-order) or the contrastmodulation depth (for second-order) of all the dots, both inside and outside the target area.

\subsection{Coherence thresholds}

The staircase controlled the number of dots within the target area that moved coherently either up or down (i.e. 'signal' dots). The second-order dots were presented at their maximum possible modulation depth $(0.8)$. The contrast of the first-order dots was set at an equal multiple of their modulation-depth threshold (approximately twice) for each observer.

\subsection{Results}

In order to aid comparison of the magnitude of effects found between the conditions when the target area location was known (fixed throughout each run of trials) to the observer and those when it was unknown (randomized on each trial), the raw data were normalized. To normalize the data, the average threshold for discriminating the direction of motion in a random, unknown position was divided by the average threshold for discriminating direction of motion in the four known positions. Fig. 2a and c show these ratios for modulation-depth thresholds and Fig. $2 b$ and $d$ show the computed ratios for the coherence thresholds.

When the motion was presented for $250 \mathrm{~ms}(\mathrm{a}, \mathrm{b})$ the ratios (of thresholds obtained in the unknown to known location) are similar, for each observer, for the luminance-modulated dots (solid bars) and the contrastmodulated dots (striped bars). This is true for both the modulation-depth thresholds (a) and the coherence thresholds (b). This is not to say that absolute performance itself was necessarily the same for the two varieties of motion stimulus, it was not and performance for contrast-defined motion was always worse, however it is the effect of knowing location that is the crucial factor of interest in this study. Once the different absolute performance levels for the two stimulus types are factored out by our normalizing procedure, the effect of not knowing the location of the coherent motion was the same for luminance-defined and contrast-modulated dots. 


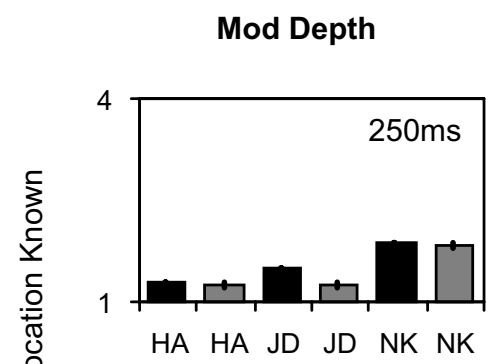

(a)

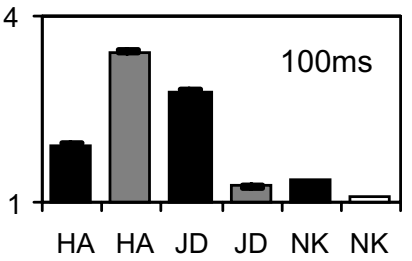

(c)

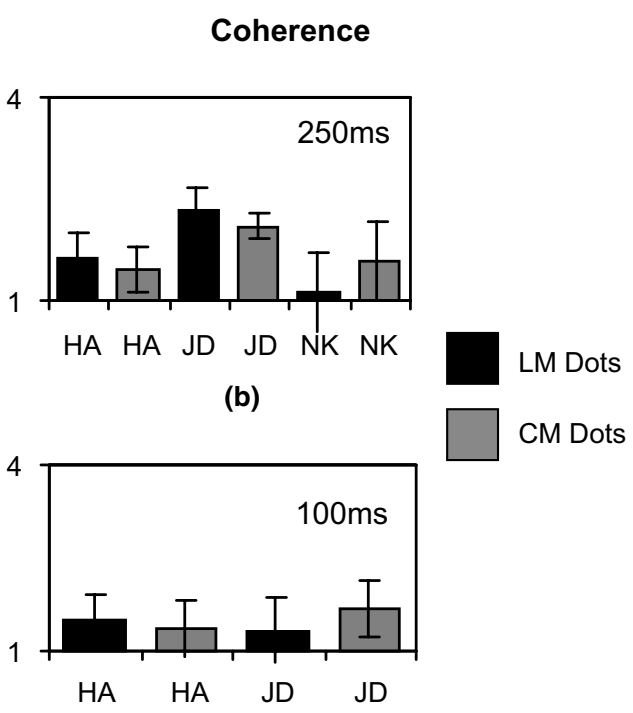

(d)

Fig. 2. Results of Experiment 1: observers discriminated the direction of motion in a target area, the prior location of which was either known or unknown. The average direction-discrimination threshold when the location was unknown was divided by the average threshold for direction discrimination in the known location to compute a threshold ratio. Performance was compared in terms of modulation-depth thresholds (a, c) and coherence thresholds $(\mathrm{b}, \mathrm{d})$ for both the luminance-modulated (LM) and contrast-modulated (CM) dots. Two stimulus durations were tested: (a, b) $250 \mathrm{~ms}$ and (c, d) $100 \mathrm{~ms}$.

When the stimulus duration was $100 \mathrm{~ms}$, the effect of not knowing the location of the motion on coherence thresholds was the same overall for luminance-modulated dots and contrast-modulated dots (d). For modulation-depth thresholds (c), one observer showed a greater effect for contrast-modulated dots (HA) but another observer showed the opposite pattern (JD). Since fixation was not monitored, it is possible that these results are due to both positional uncertainty and changes in eccentricity, despite our well trained observers and clearly visible fixation marker. Sensitivity to contrast-defined motion is lower at eccentric locations compared to sensitivity to luminance-defined stimuli. Any changes in fixation may have selectively advantaged performance with the contrast-defined stimulus, which clearly did not happen. Although the magnitude of the effect of positional uncertainty is unclear from this experiment, at present it is sufficient to conclude here that prior knowledge of stimulus location can have a marked and measurable differential effect on performance on this task. This is equally true, however, for both luminance-defined and contrast-defined motion patterns. Thus the motion of contrast-defined structure, like its luminance-defined counterpart, can be monitored simultaneously at multiple visual field positions.

\section{Experiment 2}

Experiment 1 investigated the effect of positional uncertainty solely on the ability to discriminate motion direction for both luminance-defined and contrast-defined stimuli. Although both types of motion were affected to a similar degree, we did not address the issue of observers' ability to discriminate position. In Experiment 2 observers judged the location of a motion-defined square, relative to the position of two, flanking, cue squares. This experiment was designed to measure the ability of observers to discriminate the relative location of moving contrast-modulated dots.

\subsection{Stimulus}

The stimuli were moving dots presented on a background of mean luminance. Dots were squares, subtending $0.04^{\circ}$ horizontally and vertically. First-order stimuli were typically presented with a low LM dot contrast of 0.05 (see Eq. 2) and a 2-d noise carrier added throughout the display. Second-order dots were typically presented at maximum modulation depth. 2025 dots were presented within a square stimulus display area (window) subtending 9.8 ${ }^{\circ}$. The dots moved together, coherently either left or right and with a drift speed of either 0.9 (duration $810 \mathrm{~ms}$ ) or $1.5 \%$ s (duration $540 \mathrm{~ms}$ ). Within the stimulus area two smaller squares were defined as the cue (reference) squares (each subtending $2^{\circ}$ ). These contained static dots (see the 'Introduction' and 'Results' for a further list of stimulus parameters tested in pilot studies). A central, target, square $\left(2^{\circ}\right)$ contained motion in the opposite direction to the remainder of the stimulus. The target and cue squares were defined solely by their relative motion with
463 464 465 466 467 468 469 470 471

472

473 474 475 476 477 478 479 480 481 482 483 484 485 486 487 488 489 490 491 

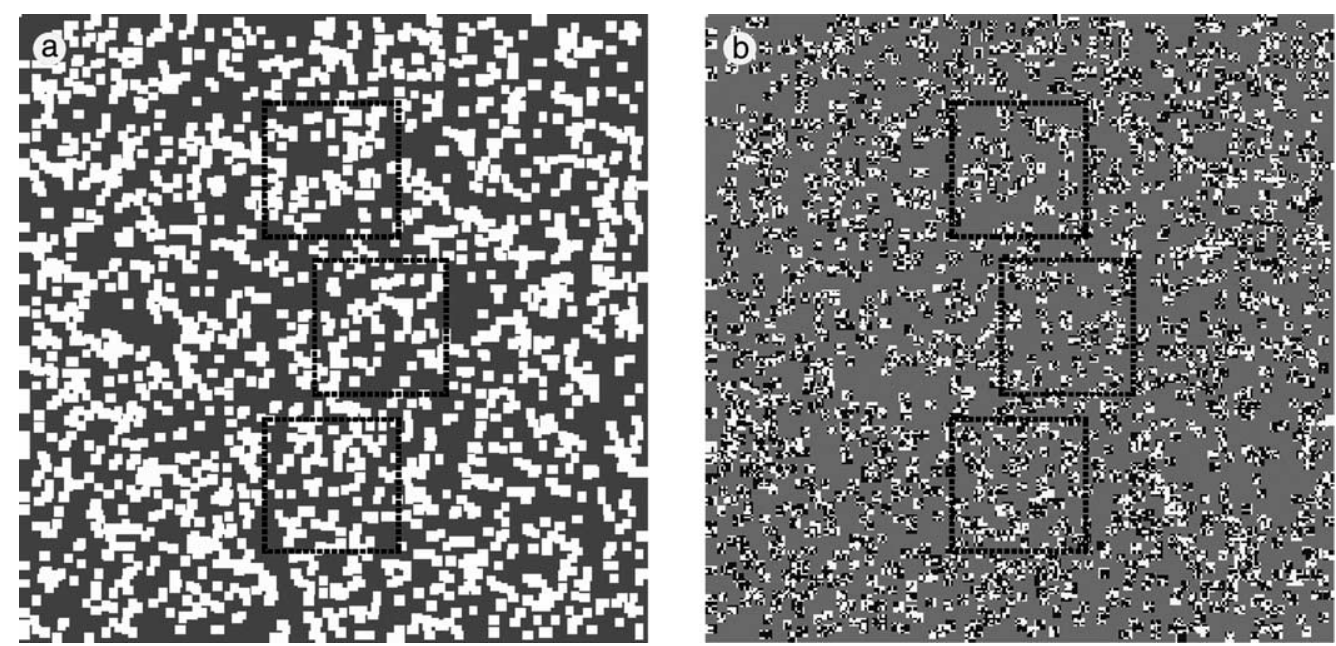

Fig. 3. (a) First-order, luminance-modulated (LM) dots used in Experiment 2. The square regions shown by the dashed outline (shown for illustrative purposes only and not visible in the actual experiments) contained motion in the opposite direction (or static dots) to the remainder of the display and were defined solely by this cue. (b) Second-order, contrast-modulated (CM) dots at maximum modulation depth as used in Experiment 2 , with square positions illustrated as in (a).

respect to the background dots. The target square was positioned in the center of the stimulus area and the cue squares were presented above and below the target square, with an edge to edge separation of $0.2^{\circ}$ (unless otherwise stated). The central, target, square was offset horizontally either to the left or right of the cue squares by a variable amount. Fig. $3 \mathrm{a}$ and $\mathrm{b}$ show illustrations of the stimuli.

\subsection{Procedure}

Observers judged, in a one interval, 2AFC procedure whether the central target square was to the left or right of the cue squares. On each trial the central square was offset to the left or right (with equal probability) by a variable amount under control of the experimenter (method of constant stimuli). Each run tested a range of offsets, spanning the entire available range. Observers indicated their response with a key press. A second key press indicated when they were ready to proceed to the next trial. A central fixation marker was presented between the trials and no feedback was given.

\subsection{Results}

Fig. 4 shows data for three observers each performing the task with 2 dot speeds (for the central, target square and background), cue squares were defined by static dots and the separation between the squares was $0.2^{\circ}$. The proportion of correct responses is plotted on the ordinate against the offset between the center and cue squares on the abscissa.

It is clear that observers rarely reached good levels of performance with either type of dot. This was the case for contrast-modulated dots (solid symbols), even though these dots were at maximum modulation depth, clearly visible and well above their motion discrimination thresholds. Performance appears to initially improve and then decrease as the offset increases. The data we show here reflect the best performance produced with contrast-modulated dot stimuli. In pilot studies we measured performance with a range of dot densities, speeds and viewing distances. In all these cases, performance was not different from chance. Observers also performed the task at lower modulation depths $(0.35)$ but performance never reached $75 \%$ correct and was close to chance. Similarly when the cue squares contained opposed motion (rather than static dots) performance was not different from chance, perhaps reflecting that it was necessary to locate both the cue and test regions. Other manipulations that might affect performance are reported below.

For low contrast luminance-modulated dots in the presence of a noise carrier (open diamonds) performance was comparable to that obtained with the contrast-modulated dots. The same ' $n$ ' shaped pattern of performance is shown. It should be noted that this pattern of performance is not an idiosyncratic feature of our particular stimulus configuration or observers. As a control, the experiment was repeated with luminancemodulated dots, but without the 2-d noise carrier. All observers reported that this task was comparatively easy. For all observers, at both speeds, offset discrimination reached $75 \%$ correct at offsets of about $0.1^{\circ}$ (see Fig. 4). Thus, the presence of an additional spatial component degraded performance for the patch of luminance-modulated dots (perhaps because it reduced its visibility).

For both the contrast-modulated patterns and the luminance-modulated patterns presented with a noise 


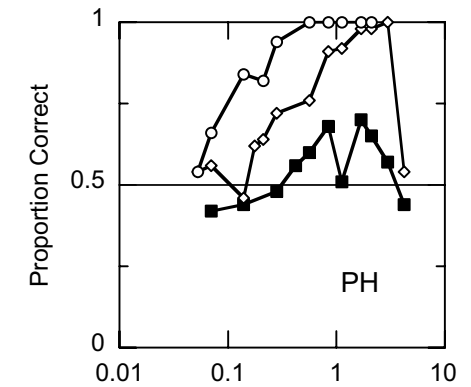

(a)

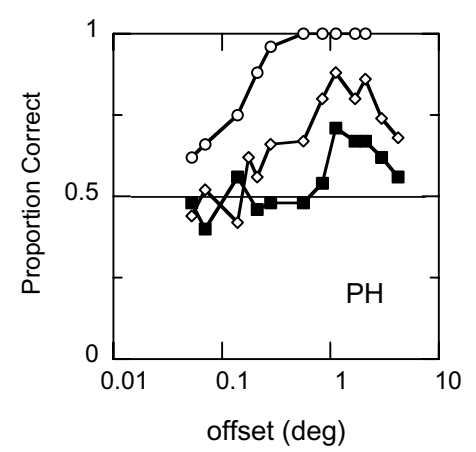

(d)

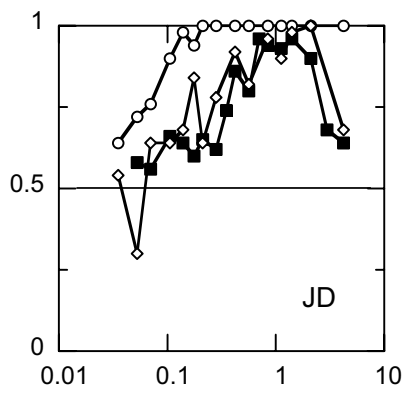

(b)

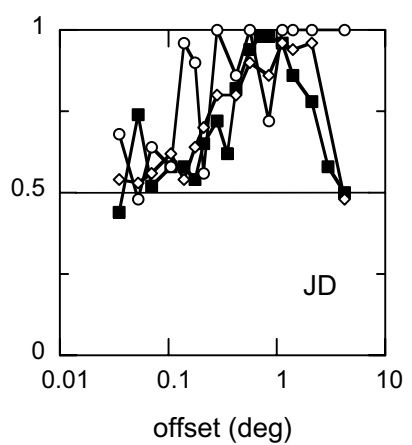

(e)

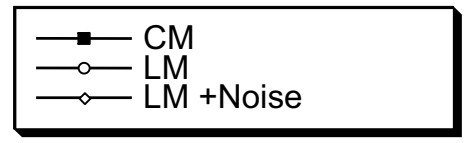

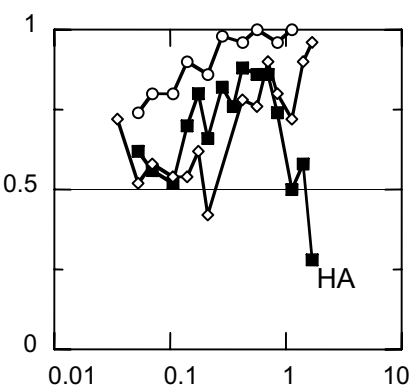

(c)

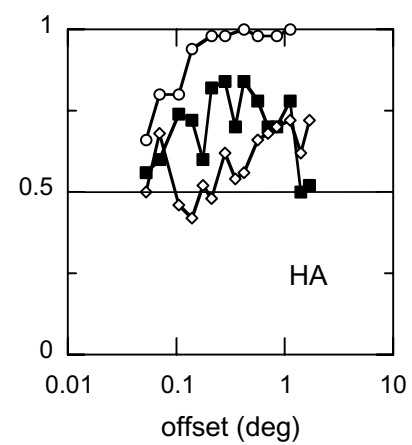

(f)

Fig. 4. Results of Experiment 2: discriminating the location (left or right of cue squares) of a motion-defined target square. The stimulus area was filled by dots moving in one direction, cue squares were defined by static dots, target squares were defined by motion in the opposite direction to the background. Dots were either contrast-modulations (CM) or luminance-modulations (LM) or luminance modulations with added visual noise. The results of three observers are shown, performing the task at two speeds: $(\mathrm{a}-\mathrm{c}) 1.5 \% \mathrm{~s}$ motion; $(\mathrm{d}-\mathrm{f}) 0.9 \% \mathrm{~s}$ motion.

mask, there is a decrease in position discrimination performance at larger offsets. Although this pattern of results has not been seen in position discrimination experiments previously, it is likely that it is a simple result of the presence of the noise pattern. At larger eccentricities the visibility of high spatial frequencies is reduced, reducing the visibility of the luminance-defined dots or reducing the visibility of the carrier of the contrast-modulations.

Since different results have, in the past been found with different separations of cue and target item (Whitaker, Bradley, Barrett, \& McGraw, 2002) we tested whether our results were specific to the configuration that we used. We increased the vertical distance between the cue squares and the target square (Fig. 5). The spatial separation between the edges of the squares was $0.2^{\circ}, 1^{\circ}$ or $2^{\circ}$. The data show that changing the separation between the squares did not change performance appreciably with the contrast-defined stimulus (shown in a-c). Similarly when luminance-defined dots were presented (shown in $\mathrm{d}-\mathrm{f}$ ), increasing the separation also had little or no effect on performance.
In the previous conditions, the cue squares were always presented in the same, central position. This was done to facilitate performance with contrast-modulated dots since pilot studies had suggested that the task was difficult. Without jittering the position of the cue squares it is not possible, however, to determine whether performance is based on the position of the target square relative to the cue squares or other cues such as the edges of the monitor. We tested the effect of randomly jittering the positions of the cue squares. The amount of jitter was randomly selected on each trial and could be between 0 and the maximum offset used in the run. Fig. 6 compares performance with and without this jitter. Jittering the position of the cue squares has little influence on performance with luminance-defined dots $(\mathrm{d}-\mathrm{f})$. For contrast-defined dots $(\mathrm{a}-\mathrm{c})$, however, adding jitter to the cue squares (solid circles) may actually marginally improve performance in some cases, though overall performance levels are again little affected by positional jittering. Thus we find no difference between contrastdefined and luminance-defined motion when it comes to indicating the position over two regions (i.e. in principle at least the task could be performed by a gross com- 


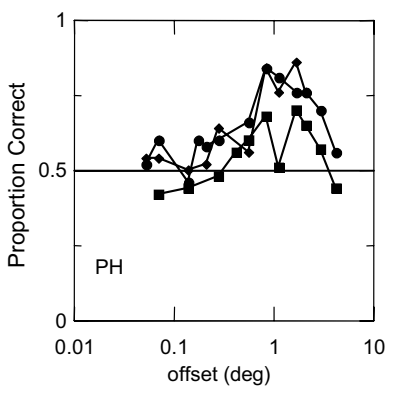

(a)

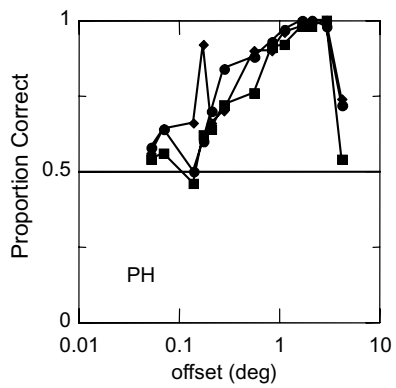

(d)

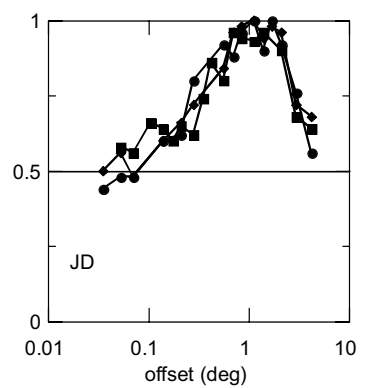

(b)

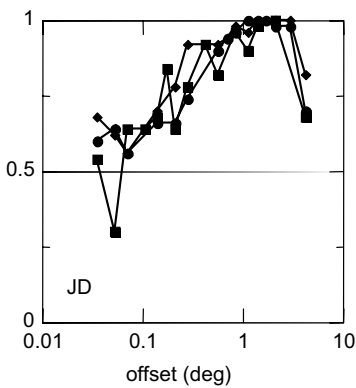

(e)

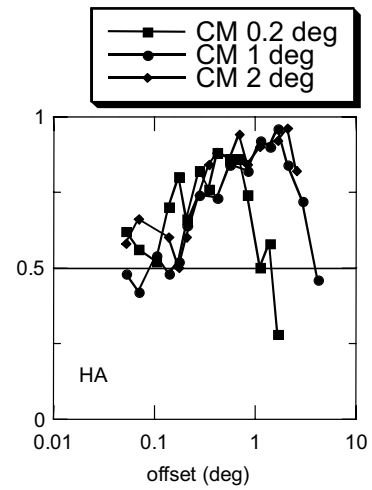

(c)

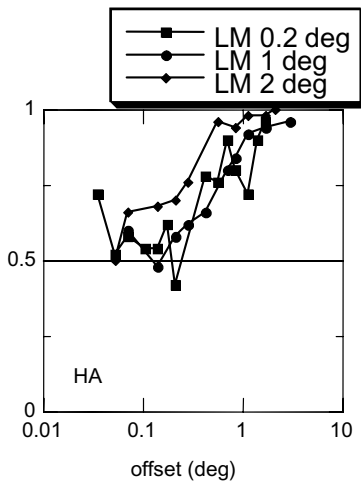

(f)

Fig. 5. Results of Experiment 2: discriminating the location (left or right of cue squares) of motion-defined target squares. Cue squares were positioned vertically at three different edge-to-edge separations from the target square (shown by the different symbols). Results from three observers are shown for $(\mathrm{a}-\mathrm{c}) \mathrm{CM}$ dots and $(\mathrm{d}-\mathrm{f}) \mathrm{LM}$ dots.

parison of the positions of the target and a single cue square) of local motion.

\section{Experiment 3}

In Experiment 1 we found that observers were able to monitor a number of the visual field locations for the presence of coherent contrast-defined motion. In Experiment 2, observers could perform a crude left-right judgment on the position of contrast-defined moving dots. Although observers performed at a comparable level with luminance-defined and contrast-defined moving dots, the stimulus conditions advantaged contrastdefined motion relative to luminance-defined motion. In the third experiment we compared the positional accuracy of luminance- and contrast-defined motion when they were equated for motion performance. To do this we compared performance at the direction-discrimination threshold for motion. Observers simultaneously judged the location and direction of motion in one of four randomly selected possible target patches containing coherent motion. We used the same stimulus configuration as previously described in Experiment 1 since our results showed that observers are able to monitor this display for both moving luminance-modulations and contrast-modulations to an equivalent degree.

\subsection{Stimuli}

Stimuli were the same as those used for the measurement of coherence thresholds in Experiment 1 with unknown location (shown schematically in Fig. 1). The presentation duration was $250 \mathrm{~ms}$ and the experiment was performed at three viewing distances of 48.5, 97.8 (as in Experiment 1) and $197 \mathrm{~cm}$. At $48.5 \mathrm{~cm}$ the display area subtended $29^{\circ}$ and the center of the target area (radius $1.7^{\circ}$ ) was at a distance of $3.5^{\circ}$ from the center of the display. At $197 \mathrm{~cm}$, the display area was $7.4^{\circ}$ in diameter and the center of the target area (radius $0.4^{\circ}$ ) was situated $0.9^{\circ}$ from the center of the display. The position of the target area was randomly chosen to be either above, below, left or right of the display center on each trial.

\subsection{Procedure}

On each trial, observers first indicated with a key press whether they perceived upwards or downwards coherent motion in a one interval, 2AFC task. Observ- 


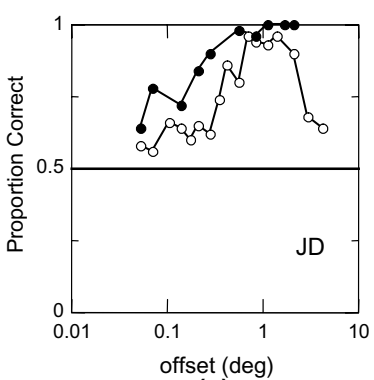

(a)

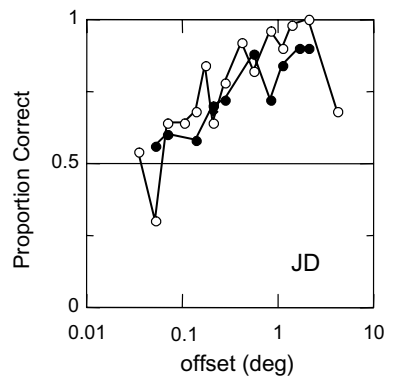

(d)

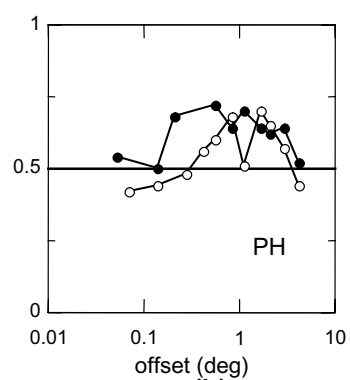

(b)

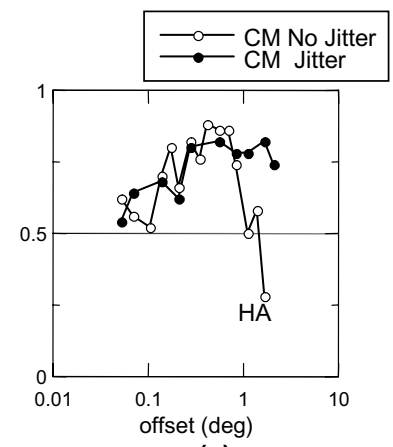

(c)

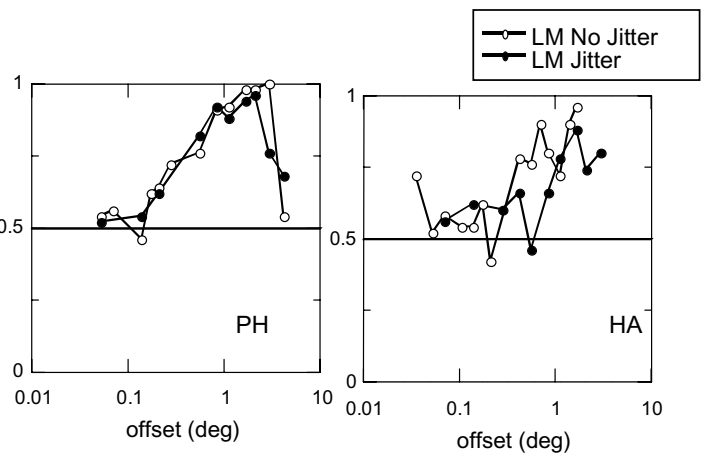

(e)

(f)

Fig. 6. Results of Experiment 2: discriminating the location (left or right of cue squares) of motion-defined target squares. Performance is shown for conditions when the cue squares remained in the same position on all trials (open symbols) and when their horizontal positions were randomly jittered on each trial (solid symbols). Results from three observers are shown with (a-c) LM dots and (d-f) CM dots.

ers then indicated, using a 4AFC procedure, whether the target area, containing coherent motion, was in the top, bottom, left or right position relative to the center of the screen. The responses from this location-discrimination task were used to control a 1-up 2-down adaptive staircase. Motion coherence within the target area was controlled by this staircase, which converged on a threshold performance level of $70 \%$. The staircase terminated after eight reversals. For each condition tested, 10 staircases were completed.

\subsection{Results}

When analyzing our results, we found that, in many conditions performance in the location-discrimination task had not reached the threshold criterion performance level. In these cases, therefore, the output of the staircase would be an unreliable and meaningless estimate of the location-identification performance of the observer. Furthermore, direction discrimination was measured in a $2 \mathrm{AFC}$ task and location-discrimination was measured using a 4AFC task. These two tasks have different chance levels (i.e. guessing rates of $50 \%$ and $25 \%$ correct, respectively) and thus percent correct performance and thresholds cannot be directly compared. To resolve these two issues we first took the raw percent correct at each stimulus level as recorded by our stair- case procedure. We averaged performance over 10 runs, but discarded any data from stimulus levels that had been tested less than 5 times (an unbiased, conservative criterion that served to minimize the impact of less reliable data points). We then normalized these data for the different guess rates of the two tasks using the following simple formula:

$P_{\mathrm{C}(\mathrm{NORM})}=\left(P_{\mathrm{C}}-G\right) /(1-G)$

where $P_{\mathrm{C}(\mathrm{NORM})}$ is the normalized proportion of correct responses at each stimulus level, $P_{\mathrm{C}}$ is the raw (unnormalized) proportion of correct responses at each stimulus level and $G$ is the task guess rate (either 0.5 or 0.25 ).

Data are shown in Figs. 7-9. In each plot the normalized proportion of correct responses is shown for the two tasks in each stimulus condition. Chance performance on both tasks is indicated as 0 , perfect performance as 1 and threshold performance (i.e. midway between perfect performance and guessing) is shown as 0.5. Each of the Figs. 7-9 shows data obtained at a different viewing distance.

At a viewing distance of $48 \mathrm{~cm}$, for luminance-modulated dots (Fig. 7a-c) the difference in performance between the two tasks is small and the functions for the two tasks overlap. For contrast-modulated dots (Fig. $7 \mathrm{~d}-\mathrm{f}$ ) observers can judge the direction of motion (solid 
(a)

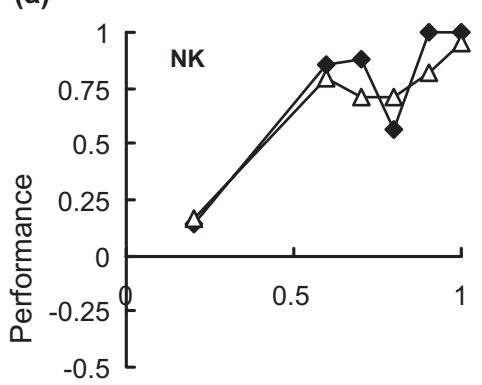

(b)

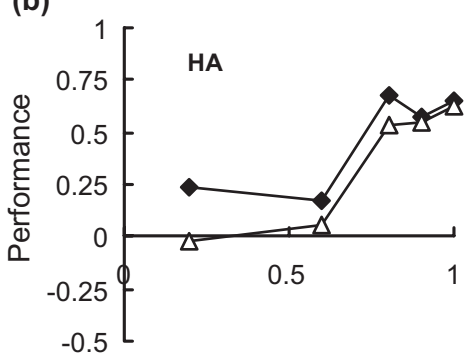

(c)

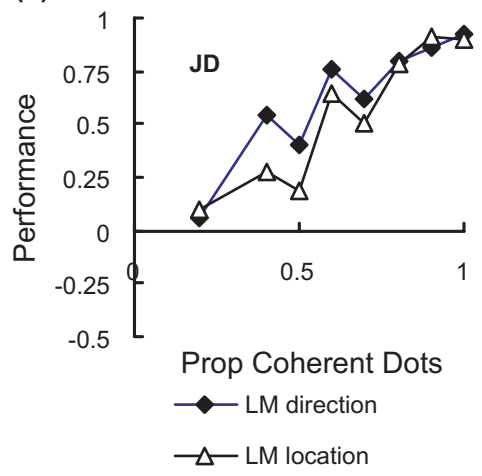

(d)

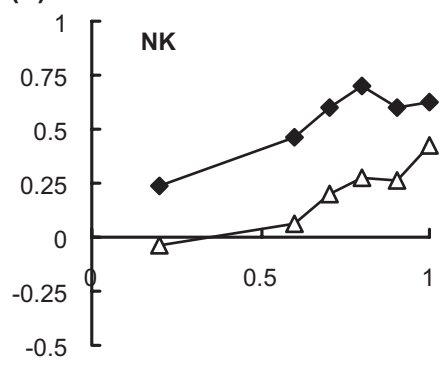

(e)

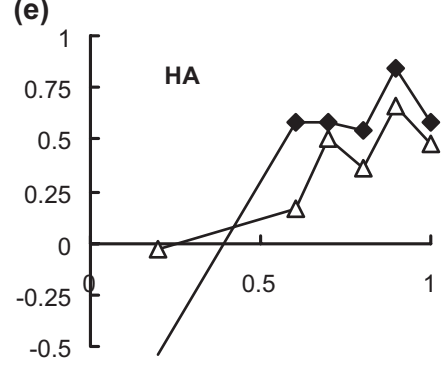

(f)

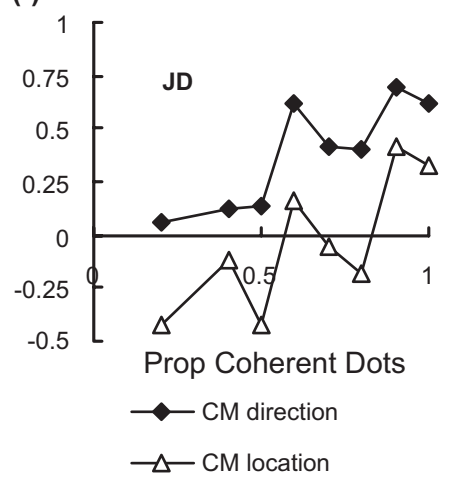

Fig. 7. Results of Experiment 3: observers judged the both the location (4AFC) and the direction (2AFC) of motion in a target area at a viewing distance of $48 \mathrm{~cm}$. Performance was normalized for the different chance levels (guessing rates) in the two tasks, such that 0 in these plots represents chance performance on both tasks and 1 represents perfect performance. Three observers performed the task with moving LM dots (a-c) and CM dots (d-f). In all cases, performance is shown for both the location discrimination (open symbols) and direction discrimination (solid symbols) tasks.

symbols) with much greater accuracy than they can judge its location (open symbols).

We tested if the difference between location-discrimination performance and direction-discrimination performance for contrast-modulated stimuli was specific to the short viewing distance. In Experiment 2, performance with contrast-defined dots decreased at the greatest eccentricities tested. In the present experiment increasing the viewing distance will decrease the eccentricity of the patches and the total stimulus area, possibly leading to an improvement in performance. At viewing distances of $97 \mathrm{~cm}$ (Fig. 8) and $194 \mathrm{~cm}$ (Fig. 9) the difference between location-discrimination performance and direction-discrimination performance is still much larger for contrast-defined motion than for luminance-defined motion. It seems that, in general, judging the location of second-order motion in one of four unpredictable locations is much more difficult than judging either the direction of that second-order motion or the location of comparable first-order motion.

To ensure that the direction-discrimination tasks were equivalent in Experiments 1 and 3, we examined the data of two observers (JD and HA) who took part in both experiments. Their psychometric functions for discriminating the direction of motion in an unknown location in Experiment 1 overlapped the psychometric functions for discriminating motion in Experiment 3. This provides good evidence that the requirement of performing two consecutive judgments in Experiment 3 (location- and direction-discrimination) rather than one (direction-discrimination) in Experiment 1, had little effect on performance and the effects found do not simply reflect a change in overall task difficulty. 
(a)

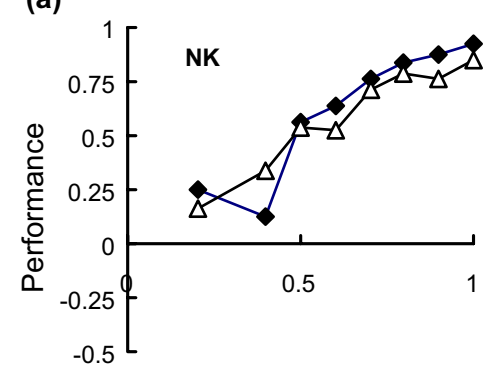

(b)

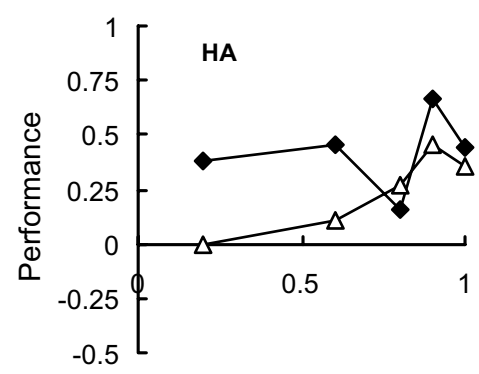

(c)

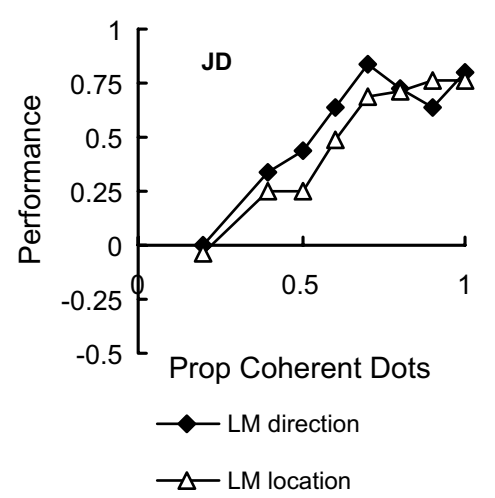

(d)

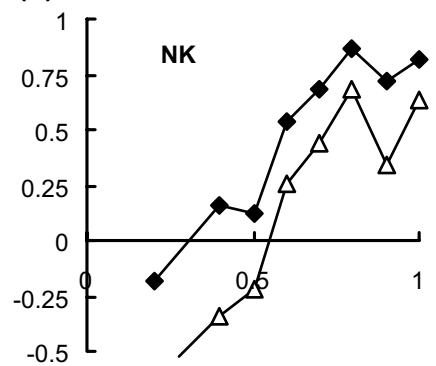

(e)

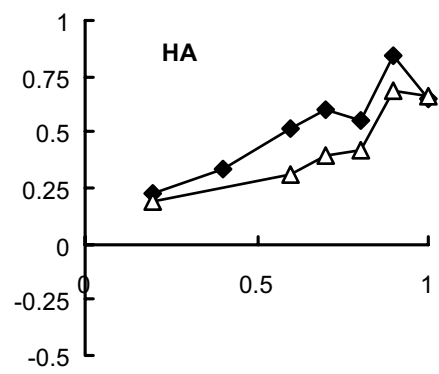

(f)

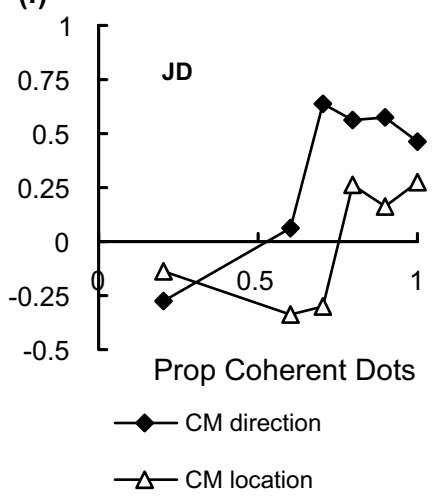

Fig. 8. Results of Experiment 3: as Fig. 7, except the viewing distance was $97 \mathrm{~cm}$.

\section{Discussion}

We investigated the limitations of the mechanism that processes contrast-defined motion, specifically with respect to encoding its position (location) in the visual field. Our motivation for this study was the previously reported failure of second-order motion to support some tasks, such as visual search and form from motion. Using contrast-defined motion as an exemplar of second-order motion we addressed two possible reasons for these failures. First, second-order motion may not be processed in an efficient, and perhaps automatic, fashion across the visual field. Second, given that the mechanisms that process second-order motion can monitor different field locations in parallel; are they also able to adequately encode the position (location) of that motion. Our results suggest that observers can monitor mechanisms for second-order motion across the visual field. The ability to locate (i.e. label position) patches of second-order motion, however, appears to be limited compared with first-order motion. It is important to emphasize that prior to formal data collection considerable effort was taken to establish the optimal conditions for measuring location-discrimination performance for the contrast-defined motion stimuli used in the current study. To achieve this we optimized a number of key stimulus parameters to obtain best performance with contrast-defined motion, including dot density, modulation depth, speed and carrier contrast. Thus we are confident that the effects found are robust and do not simply reflect a particular choice of conditions that disadvantaged contrast-defined motion.

\subsection{Monitoring second-order motion in multiple locations}

The suggestion that second-order motion is not processed efficiently over the visual field is based on the results of visual search tasks (Ashida et al., 2001) and 
(a)

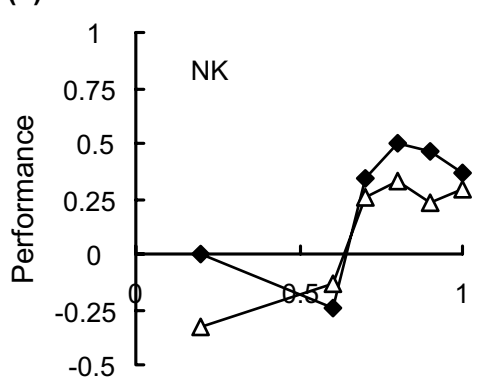

(b)

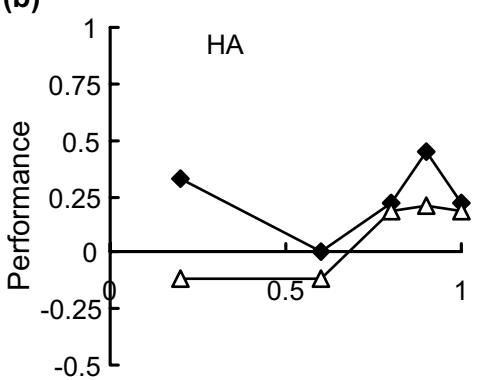

(c)

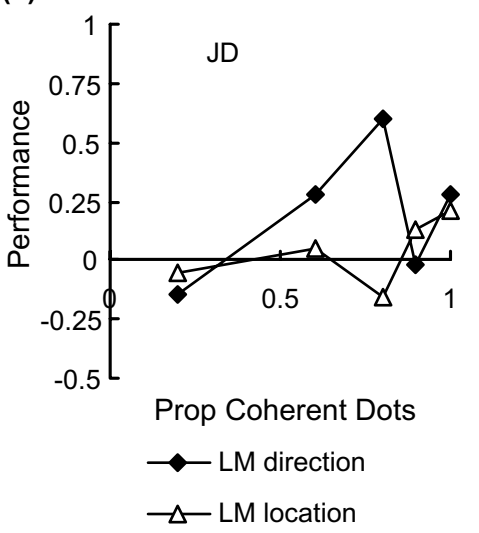

(d)

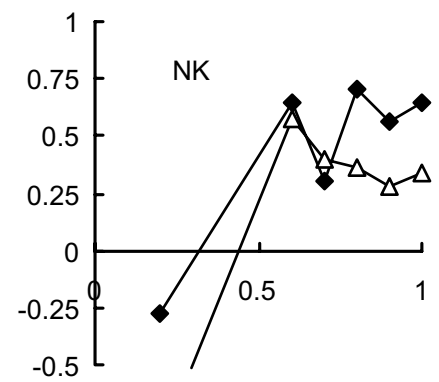

(e)

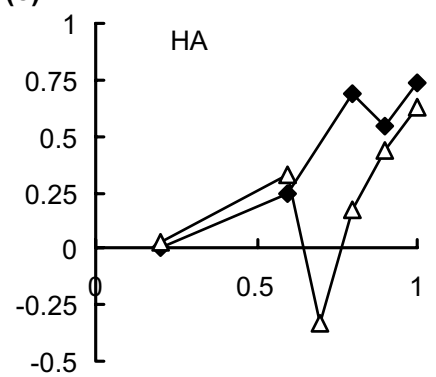

(f)

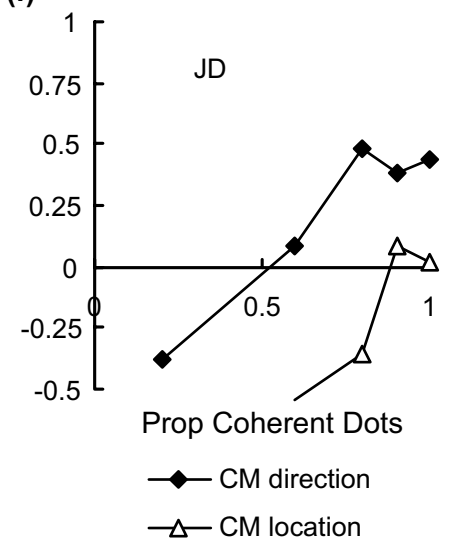

Fig. 9. Results of Experiment 3: as Fig. 7, except the viewing distance was $194 \mathrm{~cm}$.

the pattern of results found in a task where observers had to find an inconsistent direction of motion (Allen \& Derrington, 2000). In these studies the greatest effects of number of distracters were found at speeds lower than those used in Experiment 1, although similar to those used in Experiment 2. At these lower speeds, it is possible that second-order motion perception is better served by an indirect (e.g. cognitive based) higher-level mechanism (Seiffert \& Cavanagh, 1999). In Experiment 1 , the higher drift speed used would potentially favor the operation of low-level motion mechanisms that can mediate the processing of second-order motion. It appears that these mechanisms have the capacity to monitor multiple locations in the visual field.

\subsection{Position encoding for second-order motion}

We tested the fidelity with which position is encoded by the mechanisms that process contrast-defined motion in two different experiments. In Experiment 2 we tested whether these mechanisms can signal relative position over at least two regions of local motion. We found that the mechanisms that encode contrast-defined motion do not completely discard position, although good performance was highly dependant on the exact stimulus parameters used. Observers were never able to accurately discriminate position offsets as small as those typically found for luminance-defined motion stimuli. In Experiment 3 we investigated whether the mechanisms underlying luminance- and contrast-defined motion have the same positional accuracy when compared un- 
der similar levels of motion-discrimination performance. The motion coherence required for reliable position judgments was clearly higher for contrast-defined motion in Experiment 3. Thus even though we were able to show that the visual system can monitor for the presence of motion over the visual field (Experiment 1) it does not appear to encode the position of that motion with a high degree of accuracy over the same stimulus area (Experiment 3).

The underlying reason for the relatively poor position coding for contrast-defined motion is unclear. Previous studies indicate that the poor performance is not due to limitations in extracting contrast-defined spatial structure and thus it is specific to a moving contrast-defined form (Voltz \& Zanker, 1996). One possible reason is that the mechanisms that process first-order motion and those that encode second-order motion have different spatial summation areas (i.e., areas over which local motion signals are pooled or combined in order to extract the overall, net direction of movement). If a motion signal of sufficient strength falls within a directionselective detector's summation area, then that mechanism is likely to be able to signal the motion direction. Although a larger summation area would enable a motion mechanism to pool motion information over more extended regions of visual space (advantageous for encoding the net motion of large objects), it would limit the ability of that mechanism to signal the precise location of that motion. There is an inevitable trade-off between summation area extent and positional accuracy for any motion-detecting mechanism. It is thus possible, that the mechanisms that process contrast-defined motion may have larger summation areas than those that process first-order motion. Intuitively this is unsurprising since it has been found that the summation area for contrast-defined static form is larger than the summation area for similar luminance-defined form (Schofield \& Georgeson, 1999), and it is possible that this may also be true for contrast-defined motion. Similarly, the summation area for luminance-defined motion has been investigated (e.g. Anderson \& Burr, 1991; Fredericksen, Verstraten, \& vandeGrind, 1994; Watamaniuk, 1993), but it is not clear that there is yet a reliable estimate (Fredericksen, Verstraten, \& vandeGrind, 1997). There have been no studies of the summation area for secondorder, contrast-defined motion, an issue that we are currently investigating.

Contrast-defined motion might be processed by a direct, motion energy type mechanism (e.g. Lu \& Sperling, 1995) or by an indirect mechanism that relies on the change in position of image features over time (Derrington \& Ukkonen, 1999; Seiffert \& Cavanagh, 1998). Poor position acuity and larger receptive fields could be compatible with either processing mechanism. A mechanism that determines motion direction from a change in position is likely to have a receptive field that encompasses position coders at two locations. The size of the receptive field will, therefore depend on the size of the local position detectors, but will always be larger than these detectors. In the case of a direct mechanism for contrast-defined motion, it has recently been suggested that the mechanism that processes second-order motion is only weakly direction selective (Ledgeway \& Hess, 2002). This weak direction selectivity could, perhaps, arise from larger receptive fields. It is possible that both types of mechanism act on second-order motion but that in both cases position is poorly coded.

\subsection{Deficits with second-order motion}

Although we find that observers can monitor multiple locations in the visual field for the presence of a region containing coherent second-order motion, they appear to have only limited access to spatial position information. These results may explain why many previous studies have found that second-order motion is an impoverished stimulus for driving some visual phenomena. For example, the reduced performance found when judging three-dimensional shape from second-order motion might be partially attributable to poor position coding in multiple locations. Shape would be ambiguous if the exact positions of the edges that defined the shapes were poorly encoded. It is also possible that discriminating distortions in flow fields could be affected by poor position coding since these also involve accurate representation of the locations of particular velocity distributions.

Poor position coding by itself, however, may not be sufficient to explain all previously found failures with second-order motion. Slow visual search might be attributed to this deficit when the task is to locate an inconsistent motion, but performance is also poor when observers have to simply indicate the presence or absence of second-order motion in a pre-specified direction (Ashida et al., 2001). However recent evidence also suggests that the accuracy with which the direction of motion can be extracted from second-order displays is relatively poor, and these two deficits together could compromise the ability to perform visual search tasks rapidly and efficiently (Ledgeway \& Hess, 2002).

\section{Conclusion}

The mechanisms that detect contrast-defined, secondorder motion can simultaneously monitor multiple locations in the visual field for the presence of movement. It appears that the mechanism that processes second-order motion can code rudimentary spatial position to some extent, but it requires a stronger motion signal to do so and is incapable of achieving as high precision as the mechanism that processes first-order 
900 motion. The results of the present study therefore have 901 important implications for our understanding of motion 902 processing in human vision and offer some new insights 903 into why second-order motion stimuli may be relatively 904 impoverished at eliciting some visual phenomenon.

\section{Acknowledgements}

This work was supported by a Canadian Institute of Health Research Grant, MT108-18 to Robert Hess and a University of Nottingham Research Committee grant to Tim Ledgeway.

\section{References}

Albright, T. D. (1992). Form-cue invariant motion processing in primate visual cortex. Science, 255, 1141-1143.

Allen, H. A., \& Derrington, A. M. (2000). Slow discrimination of contrast-defined expansion patterns. Vision Research, 40, 735-744.

Allen, H. A., \& Derrington, A. M. (2001). Distracting attention from contrast-defined motion. Investigative Ophthalmology \& Visual Science, 42, 5061.

Anderson, S. J., \& Burr, D. C. (1991). Spatial summation properties of directionally selective mechanisms in human vision. Journal of the Optical Society of America A-Optics Image Science and Vision, 8, 1330-1339.

Ashida, H., Seiffert, A. E., \& Osaka, N. (2001). Inefficient visual search for second-order motion. Journal of the Optical Society of America A-Optics Image Science and Vision, 18, 2255-2266.

Banton, T., \& Levi, D. M. (1993). Spatial localization of motiondefined and luminance-defined contours. Vision Research, 33, 2225-2237.

Carrasco, M., Penpeci-Talgar, C., \& Eckstein, M. (2000). Spatial covert attention increases contrast sensitivity across the CSF: support for signal enhancement. Vision Research, 40, 1203-1215.

Cavanagh, P., \& Mather, G. (1989). Motion: the long and short of it. Spatial Vision, 4, 103-129.

Churan, J., \& Ilg, U. J. (2001). Processing of second-order motion stimuli in primate middle temporal area and medial superior temporal area. Journal of the Optical Society of America A-Optics Image Science and Vision, 18, 2297-2306.

Cropper, S. J. (1998). Detection of chromatic and luminance contrast modulation by the visual system. Journal of the Optical Society of America A, 15, 1969-1986.

Derrington, A. M., Badcock, D. R., \& Henning, B. (1993). Discriminating the direction of second order motion at short stimulus durations. Vision Research, 33, 1785-1794.

Derrington, A. M., \& Ukkonen, O. I. (1999). Second order motion

Dosher, B. A., Landy, M. S., \& Sperling, G. (1989). Kinetic depth effect and optic flow I: 3d shape from Fourier motion. Vision Research, 29, 1789-1813.

Foley, J. M., \& Schwarz, W. (1998). Spatial attention: effect of position uncertainty and number on distractor patterns on the thresholdsversus-contrast function for contrast discrimination. Journal of the Optical Society of America A, 15, 1036-1047.

Fredericksen, R. E., Verstraten, F. A. J., \& vandeGrind, W. A. (1994). Spatial summation and its interaction with the temporal integration mechanism in human motion perception. Vision Research, 34, 3171-3188. discrimination by feature tracking. Vision Research, 39, 1465-1475.
Fredericksen, R. E., Verstraten, F. A. J., \& vandeGrind, W. A. (1997). 956 Pitfalls in estimating motion detector receptive field geometry. 957 Vision Research, 37, 99-119.

Ho, C. E. (1998). Letter recognition reveals pathways of second order 959 and third order motion. Proceedings of the National Academy of 960 Sciences of the United States of America, 95, 400-404. 961

Kohly, R. P., \& Regan, D. (2002). Fast long-distance interactions in 962 the early processing of motion-defined form and of combinations 963 of motion-defined, luminance-defined, and cyclopean form. Vision 964 Research, 42, 969-980.

Ledgeway, T., \& Hess, R. F. (2002). Failure of direction-identification 966 for briefly presented second-order motion stimuli: evidence for 967 weak direction-selectivity of the mechanisms encoding motion. 968 Vision Research, 42, 1739-1758.

968

Ledgeway, T., \& Smith, A. T. (1994). Evidence for separate motion 970 detecting mechanisms for first and second order motion in human 971 vision. Vision Research, 34, 2727-2740.

Lu, Z., Liu, C., \& Dosher, B. A. (2000). Attention mechanisms for 973 multi-location first- and second-order motion perception. Vision 974 Research, 40, 173-186.

Lu, Z., \& Sperling, G. (1995). The functional architecture of human visual motion perception. Vision Research, 35, 2697-2722.

McCarthy, J., Pantle, A., \& Pinkus, A. (1994). Detection and discrimination performance with flicker gratings in peripheral 979 vision. Vision Research, 34, 763-773.

McGraw, P. V., Levi, D. M., \& Whitaker, D. (1999). Spatial 981 characteristics of the second order visual pathway revealed by 982 positional adaptation. Nature Neuroscience, 2, 479-484. 983

Nishida, S., Ledgeway, T., \& Edwards, M. (1997). Dual multiple-scale 984 processing for motion in the human visual system. Vision Research, 985 37, 2685-2698

Regan, D. (1986). Form from motion parallax and form from 987 luminance contrast: Vernier discrimination. Spatial Vision, 1, 988 305-318

chofield, A. J., \& Georgeson, M. A. (1999). Sensitivity to modulations of luminance and contrast in visual white noise: separate mechanisms with similar behaviour. Vision Research, 39, 2697-2716.

Schofield, A. J., \& Georgeson, M. A. (2000). The temporal properties of first and second order vision. Vision Research, 40, 2475-2487. 994

Seiffert, A. E., \& Cavanagh, P. (1998). Position displacement not 995 velocity is the cue to motion detection of second order stimuli. 996 Vision Research, 38, 3569-3582.

Seiffert, A. E., \& Cavanagh, P. (1999). Position-based motion perception for color and texture stimuli: effects of contrast and speed. Vision Research, 39, 4172-4185.

Smith, A. T., \& Ledgeway, T. (1998). Sensitivity to second order motion as a function of temporal frequency and eccentricity. Vision Research, 38, 403-410.

Voltz, H., \& Zanker, J. M. (1996). Hyperacuity for spatial localization of contrast modulated patterns. Vision Research, 36, 1329-1339.

Watamaniuk, S. N. J. (1993). Ideal observer for discrimination of the global direction of dynamic random dot stimuli. Journal of the Optical Society of America A, 10, 16-28.

Whitaker, D., Bradley, A., Barrett, B. T., \& McGraw, P. V. (2002). Isolation of stimulus characteristics contributing to Weber's law for position. Vision Research, 42, 1137-1148.

Whitaker, D., McGraw, P. V., \& Levi, D. M. (1997). The influence of adaptation on perceived visual location. Vision Research, 37, 22072216. order stimuli. Vision Research, 36, 2957-2970.

Ziegler, L. R., \& Hess, R. F. (1999). Stereoscopic depth but not shape perception from second-order stimuli. Vision Research, 39, 14911507. 